\title{
Some Fixed and Periodic Points in Abstract Metric Spaces
}

\author{
Abd Ghafur Bin Ahmad, ${ }^{1}$ Zaid Mohammed Fadail, ${ }^{1}$ \\ Mujahid Abbas, ${ }^{2}$ Zoran Kadelburg, $^{3}$ and Stojan Radenovićc \\ ${ }^{1}$ School of Mathematical Sciences, Faculty of Science and Technology, Universiti Kebangsaan Malaysia, \\ 43600 Bangi, Selangor Darul Ehsan, Malaysia \\ 2 Department of Mathematics, Lahore University of Management Sciences, Lahore 54792, Pakistan \\ ${ }^{3}$ Faculty of Mathematics, University of Belgrade, Studentski Trg 16, 11000 Beograd, Serbia \\ ${ }^{4}$ Faculty of Mechanical Engineering, University of Belgrade, Kraljice Marije 16, 11120 Beograd, Serbia
}

Correspondence should be addressed to Zaid Mohammed Fadail, zaid_fatail@yahoo.com

Received 23 July 2012; Accepted 16 September 2012

Academic Editor: Ngai-Ching Wong

Copyright (C) 2012 Abd Ghafur Bin Ahmad et al. This is an open access article distributed under the Creative Commons Attribution License, which permits unrestricted use, distribution, and reproduction in any medium, provided the original work is properly cited.

\begin{abstract}
We generalize in this paper some results on common fixed points of two, respectively four, contractive-type mappings in abstract metric spaces by removing condition of normality of the cone in their formulations. Further, some results about periodic points of self-maps are extended to the setting of abstract metric spaces.
\end{abstract}

\section{Introduction and Preliminaries}

Ordered normed spaces, cones, and topical functions have applications in applied mathematics, for instance, in using Newton's approximation method [1-4] and in optimization theory [5, 6]. $K$-metric and $K$-normed spaces were introduced in the mid-20th century ([2], see also $[3,4])$ by replacing an ordered Banach space instead of the set of real numbers, as the codomain for a metric. Huang and Zhang [7] reintroduced such spaces under the name of cone metric spaces, but went further, defining convergent and Cauchy sequences in the terms of interior points of the underlying cone. In such a way, nonnormal cones can be used as well (although they used only normal cones), paying attention to the fact that Sandwich Theorem and continuity of the metric may not hold. These and other authors (e.g., [8-14]) proved some fixed point theorems for contractive-type mappings in cone metric spaces, as well as topological vector-space-valued cone metric spaces (e.g., $[15,16])$. 18]).

The following definitions and results will be needed in the sequel (see, e.g., $[2,3,5,17$, 
Let $E$ be a real topological vector space. A subset $K$ of $E$ is called a cone if (a) $K$ is closed, nonempty and $K \neq\{\theta\}$; (b) $a, b \in \mathbb{R}, a, b \geq 0, x, y \in K$ imply that $a x+b y \in K$; (c) $K \cap(-K)=\{\theta\}$.

Given a cone $K$, we define the partial ordering $\preceq$ with respect to $K$ by $x \preceq y$ if and only if $y-x \in K$. We will write $x \ll y$ for $y-x \in$ int $K$, where int $K$ stands for the interior of $K$ and use $x \prec y$ for $(x \leq y$ and $x \neq y)$. If int $K \neq \emptyset$, then $K$ is called a solid cone [3]. Note that the notation $0 \ll c$ for an interior point of a positive cone was first used by KreIn and Rutman [19].

The cone $K$ in the topological vector space $E$ is called normal if $E$ has a base of neighborhoods of $\theta$ consisting of order-convex subsets (see [16]). In the case of a normed space, this is equivalent to the condition that there is a number $k>0$ such that, for all $x, y \in E$, $\theta \leq x \leq y$ implies $\|x\| \leq k\|y\|$. Equivalently, the cone $K$ is normal if

$$
(\forall n) \quad x_{n} \preceq y_{n} \preceq z_{n}, \quad \lim _{n \rightarrow \infty} x_{n}=\lim _{n \rightarrow \infty} z_{n}=x \text { imply } \lim _{n \rightarrow \infty} y_{n}=x
$$

For details see [5].

Example 1.1 (see [3]). Let $E=C_{\mathbb{R}}^{1}[0,1]$ with $\|x\|=\|x\|_{\infty}+\left\|x^{\prime}\right\|_{\infty}$ and $K=\{x \in E: x(t) \geq 0\}$. This cone is nonnormal. Consider, for example, $x_{n}(t)=t^{n} / n$ and $y_{n}(t)=1 / n$. Then $\theta \preceq x_{n} \leq$ $y_{n}$, and $\lim _{n \rightarrow \infty} y_{n}=\theta$, but $\left\|x_{n}\right\|=\max _{t \in[0,1]}\left|t^{n} / n\right|+\max _{t \in[0,1]}\left|t^{n-1}\right|=1 / n+1>1$; hence $x_{n}$ does not converge to zero. It follows by (1.1) that $K$ is a nonnormal cone.

Definition 1.2 (see $[4,15,16])$. Let $X$ be a nonempty set and $E$ a topological vector space with a cone $K$. Suppose that a mapping $d: X \times X \rightarrow E$ satisfies the following:

$\left(\mathrm{d}_{1}\right) \theta \preceq d(x, y)$ for all $x, y \in X$ and $d(x, y)=\theta$ if and only if $x=y$;

$\left(\mathrm{d}_{2}\right) d(x, y)=d(y, x)$ for all $x, y \in X$;

$\left(\mathrm{d}_{3}\right) d(x, z) \preceq d(x, y)+d(y, z)$ for all $x, y, z \in X$.

The function $d$ is called an abstract metric and $(X, d)$ is called an abstract metric space (or a topological vector-space-valued cone metric space or a $K$-metric space); we will use the first mentioned term.

The concept of an abstract metric space is obviously more general than that of a metric space. If $E$ is a Banach space then abstract metric space becomes a cone metric space of [7]. For new results in cone metric spaces see [20-26].

Definition 1.3. Let $(X, d)$ be an abstract metric space. We say that a sequence $\left\{x_{n}\right\}$ in $X$ is

(i) a Cauchy sequence if, for every $c$ in $E$ with $\theta \ll c$, there is an $n_{0} \in \mathbb{N}$ such that for all $m, n>n_{0}, d\left(x_{m}, x_{n}\right) \ll c ;$

(ii) a convergent sequence if, for every $c$ in $E$ with $\theta \ll c$, there is an $n_{0} \in \mathbb{N}$ such that for all $n>n_{0}, d\left(x_{n}, x\right) \ll c$ for some fixed $x \in X$.

An abstract metric space $X$ is said to be complete if every Cauchy sequence in $X$ is convergent in $X$. 
Let $(X, d)$ be an abstract metric space. The following properties are often used, particularly in the case when the underlying cone is nonnormal. The only assumption is that the cone $K$ is solid. For details about these properties see, for example, [11].

$\left(\mathrm{p}_{1}\right)$ If $a \leq h a$ where $a \in K$ and $h \in[0,1)$, then $a=\theta$.

$\left(\mathrm{p}_{2}\right)$ If $\theta \leq u \ll c$ for each $c, \theta \ll c$, then $u=\theta$.

$\left(\mathrm{p}_{3}\right)$ If $u \leq v$ and $v \ll w$, then $u \ll w$.

$\left(\mathrm{p}_{4}\right)$ If $c \in$ int $K, \theta \leq x_{n}$, and $x_{n} \rightarrow \theta$, then there exists $k \in \mathbb{N}$ such that, for all $n>k$ we have $x_{n} \ll c$. (Note that, in general, the converse is not true. Indeed, in Example 1.1, $x_{n} \nrightarrow \theta$, but $x_{n} \ll c$ for $n$ sufficiently large.)

In generalizing some theorems of Huang-Zhang [7], Abbas and Rhoades proved the following result in abstract metric spaces over normal cones.

Theorem 1.4 (see [9]). Let $(X, d)$ be a complete cone metric space over a normal cone. Suppose that $f, g: X \rightarrow X$ are two self-maps satisfying

$$
d(f x, g y) \leq \alpha d(x, y)+\beta[d(x, f x)+d(y, g y)]+\gamma[d(x, g y)+d(y, f x)]
$$

for all $x, y \in X$, where $\alpha, \beta, \gamma \geq 0$, and $\alpha+2 \beta+2 \gamma<1$. Then $f$ and $g$ have a unique common fixed point in $X$. Moreover, any fixed point of $f$ is a fixed point of $g$ and conversely.

Sing et al. extended this result of Abbas-Rhoades to four maps. They proved the following theorem.

Theorem 1.5 (see [27]). Let $(X, d)$ be a complete cone metric space over a normal cone. Suppose that the mappings $f, g, S$, and $T$ are four selfmaps on $X$ such that $f X \subset T X$ and $g X \subset S X$ and satisfying

$$
d(f x, g y) \leq \alpha d(S x, T y)+\beta[d(S x, f x)+d(T y, g y)]+\gamma[d(S x, g y)+d(T y, f x)]
$$

for all $x, y \in X$, where $\alpha, \beta, \gamma \geq 0$ and $\alpha+2 \beta+2 \gamma<1$. Suppose that the pairs $\{f, S\}$ and $\{g, T\}$ are weakly compatible. Then $f, g, S$, and $T$ have a unique common fixed point.

In 1977, Rhoades proved the following interesting result.

Theorem 1.6 (see [28]). Let $(X, d)$ be a complete metric space. Let $f: X \rightarrow X$, and suppose that there exist decreasing functions $\alpha_{i}:(0,+\infty) \rightarrow[0,1), i=1, \ldots, 5$, such that $\sum_{i=1}^{5} \alpha_{i}(t)<1$ for each $t \in(0,+\infty)$ and satisfying

$$
\begin{aligned}
d(f x, f y) \leq & \alpha_{1}(d(x, y)) d(x, y)+\alpha_{2}(d(x, y)) d(x, f x)+\alpha_{3}(d(x, y)) d(y, f y) \\
& +\alpha_{4}(d(x, y)) d(f y, x)+\alpha_{5}(d(x, y)) d(f x, y),
\end{aligned}
$$

for all $x, y \in X, x \neq y$. Then $f$ has a unique fixed point $z$ and for each $x_{0} \in X$ the sequence $\left\{f^{n} x_{0}\right\}$ converges to $z$.

We generalize in this paper Theorems 1.4 and 1.5 by removing normality condition in their formulations. An example will show that these generalizations are proper. Further, 
some results of Abbas and Rhoades about periodic points of selfmaps from [29] are extended to abstract metric spaces. Theorem 1.6 is also presented in this new setting, with a slightly shorter proof.

Note that it was shown in $[15,30,31]$ that some of the fixed point results in abstract metric spaces can be directly reduced to the respective metric results. However, the results of the present paper do not fall into this category, since some of them are new even in the context of metric spaces.

\section{Fixed Point Theorems}

In this section we will prove Theorems 1.4 and 1.5 by omitting the assumption of normality in the results. We use only the definition of convergence in terms of the relation "«". The only assumption is that $K$ is a solid cone, so we use neither continuity of the vector metric $d$, nor Sandwich Theorem. We begin with the following.

Theorem 2.1. Let $(X, d)$ be an abstract metric space over a solid cone $K$. Suppose that $f, g, S$, and $T$ are four self-maps on $X$ such that $f X \subset T X$ and $g X \subset S X$ and suppose that at least one of these four subsets of $\mathrm{X}$ is complete. Let

$$
d(f x, g y) \leq \alpha d(S x, T y)+\beta[d(S x, f x)+d(T y, g y)]+\gamma[d(S x, g y)+d(T y, f x)]
$$

for all $x, y \in X$, where $\alpha, \beta, \gamma \geq 0$ and $\alpha+2 \beta+2 \gamma<1$. Then the pairs $(f, S)$ and $(g, T)$ have a unique common point of coincidence. If, moreover, pairs $(f, S)$ and $(g, T)$ are weakly compatible, then $f, g, S$, and $T$ have a unique common fixed point.

For definitions of terms like "point of coincidence" and "weakly compatible pair" see, for example, [11].

Remark 2.2. In the papers [9] and [27], the cone $K$ is supposed to be normal and solid. In that case the proof is essentially the same as in the setting of usual metric spaces.

We now give the proof of Theorem 2.1.

Proof. Suppose $x_{0} \in X$ is an arbitrary point, and define the sequence $\left\{y_{n}\right\}$ by $y_{2 n}=f x_{2 n}=$ $T x_{2 n+1}, y_{2 n+1}=g x_{2 n+1}=S x_{2 n+2}, n=0,1,2, \ldots$ Now, as in [27], by (2.1), we have

$$
\begin{aligned}
d\left(y_{2 n}, y_{2 n+1}\right)= & d\left(f x_{2 n}, g x_{2 n+1}\right) \\
\leq & \alpha d\left(S x_{2 n}, T x_{2 n+1}\right)+\beta\left[d\left(S x_{2 n}, f x_{2 n}\right)+d\left(T x_{2 n+1}, g x_{2 n+1}\right)\right] \\
& +\gamma\left[d\left(S x_{2 n}, g x_{2 n+1}\right)+d\left(T x_{2 n+1}, f x_{2 n}\right)\right] \\
\leq & (\alpha+\beta+\gamma) d\left(y_{2 n-1}, y_{2 n}\right)+(\beta+\gamma) d\left(y_{2 n}, y_{2 n+1}\right)
\end{aligned}
$$

which implies that $d\left(y_{2 n}, y_{2 n+1}\right) \preceq \delta d\left(y_{2 n-1}, y_{2 n}\right)$, where $\delta=(\alpha+\beta+\gamma) /(1-(\beta+\gamma))<1$. Similarly it can be shown that

$$
d\left(y_{2 n+1}, y_{2 n+2}\right) \preceq \delta d\left(y_{2 n}, y_{2 n+1}\right) .
$$


Therefore, for all $n$,

$$
d\left(y_{n}, y_{n+1}\right) \leq \delta d\left(y_{n-1}, y_{n}\right) \leq \cdots \leq \delta^{n} d\left(y_{0}, y_{1}\right)
$$

Now, for any $m>n$,

$$
\begin{aligned}
d\left(y_{n}, y_{m}\right) & \leq d\left(y_{n}, y_{n+1}\right)+d\left(y_{n+1}, y_{n+2}\right)+\cdots+d\left(y_{m-1}, y_{m}\right) \\
& \leq\left[\delta^{n}+\delta^{n+1}+\cdots+\delta^{m-1}\right] d\left(y_{0}, y_{1}\right) \\
& \leq \frac{\delta^{n}}{1-\delta} d\left(y_{0}, y_{1}\right) \longrightarrow \theta \quad \text { as } n \longrightarrow \infty
\end{aligned}
$$

Thus, by properties $\left(\mathrm{p}_{4}\right)$ and $\left(\mathrm{p}_{3}\right)$ and Definition $1.3,\left\{y_{n}\right\}$ is a Cauchy sequence.

Suppose, for example, that $S X$ is a complete subset of $X$. Then $y_{n} \rightarrow u=S v, n \rightarrow \infty$, for some $v \in X$. Of course, subsequences $\left\{y_{2 n-1}\right\}$ and $\left\{y_{2 n}\right\}$ also converge to $u$. Let us prove that $f v=u$. Using (2.1) we get that

$$
\begin{aligned}
d(f v, u)= & d\left(f v, g x_{2 n-1}\right)+d\left(g x_{2 n-1}, u\right) \preceq \alpha d\left(S v, T x_{2 n-1}\right)+\beta\left[d(S v, f v)+d\left(T x_{2 n-1}, g x_{2 n-1}\right)\right] \\
& +\gamma\left[d\left(S v, g x_{2 n-1}\right)+d\left(T x_{2 n-1}, f v\right)\right]+d\left(g x_{2 n-1}, u\right)
\end{aligned}
$$

which further implies that

$$
d(f v, u) \leq \frac{\alpha+\beta+\gamma}{1-(\beta+\gamma)} d\left(u, T x_{2 n-1}\right)+\frac{1+\beta+\gamma}{1-(\beta+\gamma)} d\left(u, g x_{2 n-1}\right)
$$

Let $\theta \ll c$ be given. Since $y_{n} \rightarrow u$ as $n \rightarrow \infty$, choose a natural number $n_{0}$ such that for all $n>n_{0}$ (Definition 1.3) we have that

$$
d\left(u, T x_{2 n-1}\right) \ll \frac{1-(\beta+\gamma)}{2(\alpha+\beta+\gamma)} c, \quad d\left(u, g x_{2 n-1}\right) \ll \frac{1-(\beta+\gamma)}{2(1+\beta+\gamma)} c .
$$

Thus, according to (2.7) we obtain $d(f v, u) \ll(c / 2)+(c / 2)=c$. Therefore, $d(f v, u) \ll c$ for all $c \in$ int $K$. Using property $\left(\mathrm{p}_{2}\right)$, it follows that $d(f v, u)=\theta$ and so $f v=u=S v$. Since $u \in f X \subset T X$, we get that there exists $w \in X$ such that $T w=u$. Let us prove that also $g w=u$. By triangle inequality and (2.1), we have

$$
\begin{aligned}
d(g w, u) \leq & d\left(g w, f x_{2 n}\right)+d\left(f x_{2 n}, u\right) \\
\leq & \alpha d\left(S x_{2 n}, T w\right)+\beta\left[d\left(S x_{2 n}, f x_{2 n}\right)+d(T w, g w)\right] \\
& +\gamma\left[d\left(S x_{2 n}, g w\right)+d\left(T w, f x_{2 n}\right)\right]+d\left(g x_{2 n-1}, u\right)
\end{aligned}
$$


which further implies that

$$
d(g w, u) \leq \frac{\alpha+\beta+\gamma}{1-(\beta+\gamma)} d\left(u, S x_{2 n}\right)+\frac{1+\beta+\gamma}{1-(\beta+\gamma)} d\left(u, f x_{2 n}\right) .
$$

Now, for given $\theta \ll c$, since $y_{n} \rightarrow u$ as $n \rightarrow \infty$, choose a natural $n_{1}$ such that for all $n>n_{1}$ we have that

$$
d\left(u, S x_{2 n}\right) \ll \frac{1-(\beta+\gamma)}{2(\alpha+\beta+\gamma)} c, \quad d\left(u, f x_{2 n}\right) \ll \frac{1-(\beta+\gamma)}{2(1+\beta+\gamma)} c .
$$

According to (2.10) we obtain $d(g w, u) \ll(c / 2)+(c / 2)=c$. Therefore, $d(g w, u) \ll c$ for all $c \in$ int $K$. Using property $\left(\mathrm{p}_{2}\right)$, it follows that $d(g w, u)=\theta$ and so $g w=u=T w$. We have proved that $u$ is a common point of coincidence for pairs $(f, S)$ and $(g, T)$.

If now these pairs are weakly compatible, then $f u=f S v=S f v=S u=z_{1}$ (say) and $g u=g T w=T g w=T u=z_{2}$ (say). Moreover,

$$
\begin{aligned}
d\left(z_{1}, z_{2}\right)= & d(f u, g u) \leq \alpha d(S u, T u)+\beta[d(S u, f u)+d(T u, g u)] \\
& +\gamma[d(S u, g u)+d(T u, f u)] \\
= & \alpha d\left(z_{1}, z_{2}\right)+\beta\left[d\left(z_{1}, z_{1}\right)+d\left(z_{2}, z_{2}\right)\right]+\gamma\left[d\left(z_{1}, z_{2}\right)+d\left(z_{2}, z_{1}\right)\right] \\
= & (\alpha+2 \gamma) d\left(z_{1}, z_{2}\right) \leq(\alpha+\beta+\gamma) d\left(z_{1}, z_{2}\right) \prec d\left(z_{1}, z_{2}\right)
\end{aligned}
$$

implies that $z_{1}=z_{2}$. So we have that $f u=g u=S u=T u$. It remains to prove that, for example, $u=g u$. Indeed,

$$
\begin{aligned}
d(u, g u)= & d(f v, g u) \leq \alpha d(S v, T u)+\beta[d(S v, f v)+d(T u, g u)] \\
& +\gamma[d(S v, g u)+d(T u, f v)] \\
= & \alpha d(u, g u)+\beta[\theta+\theta]+\gamma[d(u, g u)+d(g u, u)] \\
= & (\alpha+2 \gamma) d(u, g u) \leq(\alpha+\beta+\gamma) d(u, g u) \prec d(u, g u),
\end{aligned}
$$

implying that $g u=u$. The uniqueness follows from (2.1). The proofs for cases in which $f X$, $g X$, or $T X$ is complete are similar and are therefore omitted. The theorem is proved.

We present now two examples showing that Theorem 2.1 is a proper extension of the known results. In both examples, the conditions of Theorem 2.1 are fulfilled, but in the first one (because of nonnormality of the cone) the main theorems from $[9,27]$ cannot be applied.

Example 2.3 (the case of a nonnormal cone). Let $X=[0,1]$, and let $E$ be the set of all realvalued functions on $X$ which also have continuous derivatives on $X$. Note that $E$ is a vector space over $\mathbb{R}$ under usual function operations. Let $\tau$ be the strongest vector (locally convex) topology on $E$. Then $(E, \tau)$ is a topological vector space which is not normable and is not even metrizable. Let $K=\{\varphi \in E: \varphi(t) \geq 0, t \in \mathbb{R}\}$ and $d: X \times X \rightarrow E$ be defined by 
$d(x, y)(t)=|x-y| \cdot e^{t}$. Then $(X, d)$ is an abstract metric space over a nonnormal solid cone (Example 1.1). Consider the four mappings $f, g, T, S: X \rightarrow X$ defined by

$$
f x=\frac{x}{8}, \quad g x=\frac{x}{12}, \quad T x=\frac{x}{2}, \quad S x=\frac{x}{3} .
$$

Clearly $f(X) \subset T(X)$ and $g(X) \subset S(X)$.

For $x, y \in X$,

$$
\begin{gathered}
d(f x, g y)(t)=\left|\frac{x}{8}-\frac{y}{12}\right| e^{t}=\frac{1}{8}\left|x-\frac{2 y}{3}\right| e^{t}, \\
d(S x, T y)(t)=\left|\frac{x}{3}-\frac{y}{2}\right| e^{t}, \\
d(f x, S x)(t)+d(g y, T y)(t)=\left|\frac{x}{8}-\frac{x}{3}\right| e^{t}+\left|\frac{y}{12}-\frac{y}{2}\right| e^{t}=\left(\frac{5 x}{24}+\frac{5 y}{12}\right) e^{t}, \\
d(f x, T y)+d(g y, S x)(t)=\left(\left|\frac{x}{8}-\frac{y}{2}\right|+\left|\frac{y}{12}-\frac{x}{3}\right|\right) e^{t} .
\end{gathered}
$$

Now

$$
\begin{aligned}
d(f x, g y)(t)= & \frac{1}{8}\left|x-\frac{2 y}{3}\right| e^{t} \\
\leq & \frac{1}{6}\left|\frac{x}{3}-\frac{y}{2}\right| e^{t}+\frac{1}{6}\left(\frac{5 x}{24}+\frac{5 y}{12}\right) e^{t}+\frac{1}{6}\left(\left|\frac{x}{8}-\frac{y}{2}\right|+\left|\frac{y}{12}-\frac{x}{3}\right|\right) e^{t} \\
= & \alpha d(S x, T y)(t)+\beta[d(f x, S x)(t)+r d(g y, T y)(t)] \\
& +\gamma[d(f x, T y)(t)+d(g y, S x)(t)] .
\end{aligned}
$$

Thus all the conditions of Theorem 2.1 are satisfied with $\alpha+2 \beta+2 \gamma=5 / 6<1$. Note that 0 is the unique common fixed point of $f, g, S$, and $T$.

Example 2.4 (the case of a normal cone). Let $E=C_{\mathbb{R}}[0,1]$ with $K=\{\varphi \in E: \varphi(t) \geq 0, t \in[0,1]\}$ (this cone is normal; see [5]). Let $X=[0, \infty)$, and let $d: X \times X \rightarrow E$ be defined as $d(x, y)(t):=$ $|x-y| e^{t}$. Take the functions $f x=x / 3, g x=0, S X=T X=x$ which map the set $X$ into $X$. All the conditions of Theorem 2.1 are fulfilled with $\gamma=1 / 3, \alpha+2 \beta<1 / 3$. Obviously, $f, g, S$, and $T$ have the unique common fixed point $x=0$.

Remark 2.5. Taking $S=T=i_{X}$ and appropriate choices of $f, g, \alpha, \beta$, and $\gamma$ in Theorem 2.1, one easily gets [9, Corollaries 2.2-2.8]. In each of the following cases $(1)-(7),(X, d)$ is a complete abstract metric space, $K$ a solid cone, and $f$ is a selfmap on $X$.

(1) Let

$$
d\left(f^{p} x, f^{q} y\right) \leq \alpha d(x, y)+\beta\left[d\left(x, f^{p} x\right)+d\left(y, f^{q} y\right)\right]+\gamma\left[d\left(x, f^{q} y\right)+d\left(y, f^{p} x\right)\right]
$$


for all $x, y \in X$, where $\alpha, \beta, \gamma \geq 0$ and $\alpha+2 \beta+2 \gamma<1$, and $p$ and $q$ are fixed positive integers. Then $f$ has a unique fixed point in $X$.

(2) If

$$
d(f x, f y) \leq \alpha d(x, y)+\beta[d(x, f x)+d(y, f y)]+\gamma[d(x, f y)+d(y, f x)]
$$

for all $x, y \in X$, where $\alpha, \beta, \gamma \geq 0$ and $\alpha+2 \beta+2 \gamma<1$, then $f$ has a unique fixed point in $X$.

(3) If

$d(f x, f y) \leq a_{1} d(x, y)+a_{2} d(x, f x)+a_{3} d(y, f y)+a_{4} d(x, f y)+a_{5} d(y, f x)$

for all $x, y \in X$, where $a_{i} \geq 0$ for each $i \in\{1,2, \ldots, 5\}$ and $a_{1}+a_{2}+\cdots+a_{5}<1$, then $f$ has a unique fixed point in $X$.

(4) If

$$
d(f x, f y) \preceq \alpha d(x, y)
$$

for all $x, y \in X$, where $\alpha \in[0,1)$, then $f$ has a unique fixed point in $X$.

(5) If

$$
d(f x, f y) \leq \beta[d(x, f x)+d(y, f y)]
$$

for all $x, y \in X$, where $\beta \in[0,1 / 2)$, then $f$ has a unique fixed point in $X$.

(6) If

$$
d(f x, f y) \preceq \gamma[d(x, f y)+d(y, f x)]
$$

for all $x, y \in X$, where $\gamma \in[0,1 / 2)$, then $f$ has a unique fixed point in $X$.

(7) If

$$
d(f x, f y) \leq \alpha d(x, y)+\beta[d(x, f x)+d(y, f y)]
$$

for all $x, y \in X$, where $\alpha, \beta \geq 0$ and $\alpha+2 \beta<1$, then $f$ has a unique fixed point in $X$. space.

We add an example of a Banach-type contraction on a nonnormal abstract metric

Example 2.6. Let $X=[0,1], E=C_{R}^{1}[0,1], K=\{\varphi \in E: \varphi(t) \geq 0\}$. An abstract metric $d$ on $X$ is defined by $d(x, y)(t):=|x-y| \cdot \phi(t)$ where $\phi \in K$ is an arbitrary function (e.g., $\left.\phi(t)=2^{t}\right)$. It is easy to see that $(X, d)$ is a complete abstract metric space. Suppose that mapping $f: X \rightarrow X$ satisfies

$$
d(f x, f y) \leq \lambda d(x, y)
$$


for all $x, y \in X$, where $\lambda \in[0,1)$. All the conditions from Remark 2.5(4) hold, and $f$ has a unique fixed point in $X$.

This example verifies that Theorem 2.1 is a proper extension of the results from [7]. Indeed, we know (see Example 1.1) that the cone $K$ is nonnormal. So, in this example Theorem 1 from [7] cannot be applied.

Corollary 2.7. Let $(X, d)$ be an abstract metric space and $K$ a solid cone. Suppose that the mappings $f, g, S$ and $T$ are four selfmaps of $X$ such that $f(X) \subset T(X)$ and $g(X) \subset S(X)$, and suppose that at least one of these four subsets of $\mathrm{X}$ is complete. Let

$$
d\left(f^{n} x, g^{n} y\right) \preceq \alpha d(S x, T y)
$$

for all $x, y \in X$, where $\alpha \in[0,1)$ and $(f, S)$ and $(g, T)$ commute. Then $f, g, S$, and $T$ have a unique common fixed point.

Proof. By Theorem 2.1, we obtain $u \in X$ such that

$$
f^{n} u=g^{n} u=S u=T u=u .
$$

The result then follows from the fact that

$$
d(f u, g u)=d\left(f^{n} f u, g^{n} g u\right) \preceq \alpha d(S f u, T g u)=\alpha d(f u, g u)
$$

since $\alpha<1$ so that $f u=g u$ by property $\left(\mathrm{p}_{1}\right)$. Again

$$
d(f u, u)=d\left(f^{n} f u, g^{n} u\right) \preceq \alpha d(S f u, T u)=\alpha d(f u, u)
$$

implies that $f u=u$. And hence $u$ is the unique common fixed point of $f, g, S$, and $T$.

\section{Periodic Point Theorems}

It is clear that if $f$ is a map which has a fixed point $p$, then $p$ is also a fixed point of $f^{n}$ for every $n \in \mathbb{N}$. However the converse is not true. For example, consider $X=\mathbb{R}$ and $f$ defined by $f x=a-x, a \neq 0$. Then $f$ has a unique fixed point at $a / 2$, but every even iterate of $f$ is the identity map, which has each point of $\mathbb{R}$ as its fixed point. On the other hand, if $X=[0,+\infty)$, $f x=x^{2}$, then every iterate of $f$ has the same fixed point as $f$. If a map $f$ satisfies $F(f)=F\left(f^{n}\right)$ for each $n \in \mathbb{N}$, where $F(f)$ stands for the set of all fixed points of $f$, then it is said to have property $P$ [29]. We will say that $f, g: X \rightarrow X$ have property $Q$ if $F(f) \cap F(g)=F\left(f^{n}\right) \cap F\left(g^{n}\right)$ for each $n \in \mathbb{N}$.

The next result is a generalization of the corresponding result in metric spaces (see [29, Theorem 1.1]). It will be deduced also without using normality of the cone.

Theorem 3.1. Let $(X, d)$ be an abstract metric space over a solid cone $K$, and let $f: X \rightarrow X$ be such that $F(f) \neq \emptyset$ and that

$$
d\left(f x, f^{2} x\right) \leq \lambda d(x, f x)
$$


holds for some $\lambda \in(0,1)$ and either (i) for all $x \in X$ or (ii) for all $x \in X, x \neq f x$. Then $f$ has property $P$.

Proof. We will always assume that $n>1$, since the statement for $n=1$ is trivial. Let $u \in F\left(f^{n}\right)$. Suppose that $f$ satisfies (i). Then

$$
\begin{aligned}
d(u, f u) & =d\left(f f^{n-1} u, f^{2} f^{n-1} u\right) \preceq \lambda d\left(f^{n-1} u, f^{n} u\right)=\lambda d\left(f f^{n-2} u, f^{2} f^{n-2} u\right) \\
& \leq \lambda^{2} d\left(f^{n-2} u, f^{n-1} u\right) \preceq \cdots \preceq \lambda^{n} d(u, f u) \leq \lambda d(u, f u) .
\end{aligned}
$$

According to property $\left(\mathrm{p}_{1}\right)$ it follows that $d(u, f u)=\theta$, that is, $f u=u$. Suppose that $f$ satisfies (ii). If $f u=u$, then there is nothing to prove. Suppose, if possible, that $f u \neq u$. Then, similarly as in case (i) we get that $d(u, f u)=d\left(f f^{n-1} u, f^{2} f^{n-1} u\right)$. In order to use (3.1) we need that $f^{n-1} u \neq f f^{n-1} u=f^{n} u$. But, if this is not the case, then $f^{n-1} u=u$ and so $u=f^{n} u=f u$, a contradiction. Hence, applying (3.1) we obtain that

$$
d(u, f u)=d\left(f f^{n-1} u, f^{2} f^{n-1} u\right) \leq \lambda d\left(f^{n-1} u, f^{n} u\right)=\lambda d\left(f f^{n-2} u, f^{2} f^{n-2} u\right) .
$$

Repeating the same argument several times we finally obtain, similarly as in case (i), that $d(u, f u) \leq \lambda^{n} d(u, f u)$, which again implies $u=f u$ since $\lambda \in(0,1)$, a contradiction.

Corollary 3.2. Let $(X, d)$ be an abstract metric space over a solid cone $K$. Suppose that a mapping $f: X \rightarrow X$ satisfies

$$
d(f x, f y) \leq \alpha d(x, y)+\beta[d(x, f x)+d(y, f y)]+\gamma[d(x, f y)+d(y, f x)]
$$

for all $x, y \in X$, where $\alpha, \beta, \gamma \geq 0$ and $\alpha+2 \beta+2 \gamma<1$. Then $f$ has property $P$.

Proof. From Remark 2.5(2), $F(f) \neq \emptyset$. We will prove that $f$ satisfies the condition (i) of Theorem 3.1. Indeed,

$$
\begin{aligned}
d\left(f x, f^{2} x\right)= & d(f x, f f x) \leq \alpha d(x, f x)+\beta\left[d(x, f x)+d\left(f x, f^{2} x\right)\right] \\
& +\gamma\left[d\left(x, f^{2} x\right)+d(f x, f x)\right] \\
\leq & \alpha d(x, f x)+\beta\left[d(x, f x)+d\left(f x, f^{2} x\right)\right]+\gamma\left[d(x, f x)+d\left(f x, f^{2} x\right)\right],
\end{aligned}
$$

which implies that $d\left(f x, f^{2} x\right) \leq \lambda d(x, f x)$, where $\lambda=(\alpha+\beta+\gamma) /(1-(\beta+\gamma))<1$. Hence, $f$ has property $P$.

The method of proof of the following result differs to the one from [29] (see also [9, Theorem 3.2]).

Theorem 3.3. Let $(X, d)$ be a complete abstract metric space over a solid cone K. Suppose that mappings $f, g: X \rightarrow X$ satisfy (2.1) (with $S=T=i_{X}$ ). Then $f$ and $g$ have property $Q$. 
Proof. By Theorem 2.1, we have that $F(f) \cap F(g)=\{u\}$, where $u$ is the unique common fixed point of $f$ and $g$. So $F\left(f^{n}\right) \cap F\left(g^{n}\right) \neq \emptyset$ for each $n \in \mathbb{N}$. Let $v \in F\left(f^{n}\right) \cap F\left(g^{n}\right)$, where $n>1$ is arbitrary. Then, we obtain

$$
\begin{aligned}
d(u, v)= & d\left(f^{n} u, g^{n} v\right)=d\left(f f^{n-1} u, g g^{n-1} v\right) \\
\leq & \alpha d\left(f^{n-1} u, g^{n-1} v\right)+\beta\left[d\left(f^{n-1} u, f^{n} u\right)+d\left(g^{n-1} v, g^{n} v\right)\right] \\
& +\gamma\left[d\left(f^{n-1} u, g^{n} v\right)+d\left(g^{n-1} v, f^{n} u\right)\right] \\
= & \alpha d\left(u, g^{n-1} v\right)+\beta d\left(g^{n-1} v, g^{n} v\right)+\gamma\left[d\left(u, g^{n} v\right)+d\left(u, g^{n-1} v\right)\right] \\
\leq & \alpha d\left(u, g^{n-1} v\right)+\beta d\left(g^{n-1} v, u\right)+\beta d\left(u, g^{n} v\right)+\gamma\left[d\left(u, g^{n} v\right)+d\left(u, g^{n-1} v\right)\right],
\end{aligned}
$$

wherefrom it follows that $d\left(u, g^{n} v\right) \preceq \delta d\left(u, g^{n-1} v\right)$, where $\delta=(\alpha+\beta+\gamma) /(1-(\beta+\gamma))$.

Further, we have that

$$
d\left(u, g^{n-1} v\right) \leq \delta d\left(u, g^{n-2} v\right)
$$

Indeed,

$$
\begin{aligned}
d\left(u, g^{n-1} v\right)= & d\left(f^{n-1} u, g^{n-1} v\right)=d\left(f f^{n-2} u, g g^{n-2} v\right) \\
\leq & \alpha d\left(f^{n-2} u, g^{n-2} v\right)+\beta\left[d\left(f^{n-2} u, f^{n-1} u\right)+d\left(g^{n-2} v, g^{n-1} v\right)\right] \\
& +\gamma\left[d\left(f^{n-2} u, g^{n-1} v\right)+d\left(g^{n-2} v, f^{n-1} u\right)\right] \\
= & \alpha d\left(u, g^{n-2} v\right)+\beta d\left(g^{n-2} v, g^{n-1} v\right)+\gamma\left[d\left(u, g^{n-1} v\right)+d\left(u, g^{n-2} v\right)\right] \\
\leq & \alpha d\left(u, g^{n-2} v\right)+\beta d\left(g^{n-2} v, u\right)+\beta d\left(u, g^{n-1} v\right) \\
& +\gamma\left[d\left(u, g^{n-1} v\right)+d\left(u, g^{n-2} v\right)\right],
\end{aligned}
$$

which implies (3.7). Hence,

$$
d(u, v)=d\left(u, g^{n} v\right) \leq \delta d\left(u, g^{n-1} v\right) \leq \delta^{2} d\left(u, g^{n-2} v\right) \leq \cdots \leq \delta^{n} d(u, v) .
$$

Since $\delta^{n} \in[0,1)$, according to property $\left(\mathrm{p}_{1}\right)$ it follows $d(u, v)=\theta$. Hence $v=u$, which implies that $f$ and $g$ have property $Q$.

Corollary 3.4. Let $(X, d)$ be a complete abstract metric space over a solid cone $K$. Suppose that the mapping $f: X \rightarrow X$ satisfies one of the conditions (3)-(6) of Remark 2.5. Then $f$ has property P. 
Remark 3.5. In the paper [9], the space $(X, d)$ is supposed to be a complete cone metric space over a normal and solid cone $K$. Hence, our Theorems 3.1, 3.3, and Corollary 3.2 are proper extensions of Theorems 3.1, 3.2. and 3.3 from [9]. e.g., [5]).

In the following result the cone $K$ is regular, hence also normal (for the definition see,

Theorem 3.6. Let $(X, d)$ be an abstract metric space over a regular cone $K$. Let $f, g: X \rightarrow X$ be two mappings such that $f X \subset g X$ and one of these subset of $X$ is complete. Suppose that there exist decreasing functions $\alpha_{i}: K \rightarrow[0,1), i=1, \ldots, 5$, such that $\sum_{i=1}^{5} \alpha_{i}(t)<1$ for each $t \in K$ and satisfying

$$
\begin{aligned}
d(f x, f y) \leq & \alpha_{1}(d(g x, g y)) d(g x, g y)+\alpha_{2}(d(g x, g y)) d(g x, f x)+\alpha_{3}(d(g x, g y)) d(g y, f y) \\
& +\alpha_{4}(d(g x, g y)) d(f y, g x)+\alpha_{5}(d(g x, g y)) d(f x, g y)
\end{aligned}
$$

for all $x, y \in X, x \neq y$. Then $f$ and $g$ have a unique point of coincidence. If, moreover, the pair $(f, g)$ is weakly compatible, then $f$ and $g$ have a unique common fixed point.

Proof. Suppose, for example, that $g X$ is complete. Take an arbitrary $x_{0} \in X$ and, using that $f X \subset g X$, construct a Jungck sequence $\left\{y_{n}\right\}$ defined by $y_{n}=f x_{n}=g x_{n+1}, n=0,1,2, \ldots$ Let us prove that this is a Cauchy sequence. If $y_{n}=y_{n-1}$ for some $n$, then it is easy to prove that the sequence $\left\{y_{n}\right\}$ becomes eventually constant and so convergent.

Suppose that $y_{n} \neq y_{n-1}$ for each $n \in \mathbb{N}$. Using (3.10), we obtain that

$$
\begin{aligned}
d\left(y_{n}, y_{n+1}\right)= & d\left(f x_{n}, f x_{n+1}\right) \\
\leq & \alpha_{1}\left(d\left(y_{n-1}, y_{n}\right)\right) d\left(y_{n-1}, y_{n}\right)+\alpha_{2}\left(d\left(y_{n-1}, y_{n}\right)\right) d\left(y_{n-1}, y_{n}\right) \\
& +\alpha_{3}\left(d\left(y_{n-1}, y_{n}\right)\right) d\left(y_{n}, y_{n+1}\right)+\alpha_{4}\left(d\left(y_{n-1}, y_{n}\right)\right) d\left(y_{n+1}, y_{n-1}\right) \\
& +\alpha_{5}\left(d\left(y_{n-1}, y_{n}\right)\right) d\left(y_{n}, y_{n}\right),
\end{aligned}
$$

for each $n \in \mathbb{N}$. Also,

$$
\begin{aligned}
d\left(y_{n+1}, y_{n}\right)= & d\left(f x_{n}, f x_{n+1}\right) \\
\leq & \alpha_{1}\left(d\left(y_{n-1}, y_{n}\right)\right) d\left(y_{n}, y_{n-1}\right)+\alpha_{2}\left(d\left(y_{n-1}, y_{n}\right)\right) d\left(y_{n+1}, y_{n}\right) \\
& +\alpha_{3}\left(d\left(y_{n-1}, y_{n}\right)\right) d\left(y_{n-1}, y_{n}\right)+\alpha_{4}\left(d\left(y_{n-1}, y_{n}\right)\right) d\left(y_{n}, y_{n}\right) \\
& +\alpha_{5}\left(d\left(y_{n-1}, y_{n}\right)\right) d\left(y_{n+1}, y_{n-1}\right) .
\end{aligned}
$$

Adding the last two relations (and putting temporarily $\left.\alpha_{i}=\alpha_{i}\left(d\left(y_{n-1}, y_{n}\right)\right), i=1, \ldots, 5\right)$ we obtain

$$
d\left(y_{n}, y_{n+1}\right) \leq \beta\left(d\left(y_{n-1}, y_{n}\right)\right) d\left(y_{n-1}, y_{n}\right),
$$


where

$$
\beta(t)=\frac{2 \alpha_{1}(t)+\alpha_{2}(t)+\alpha_{3}(t)+\alpha_{4}(t)+\alpha_{5}(t)}{2-\left(\alpha_{2}(t)+\alpha_{3}(t)+\alpha_{4}(t)+\alpha_{5}(t)\right)} .
$$

It is easy to see that monotonicity of all $\alpha_{i}$ 's implies that $\beta$ is also a decreasing function and that $0<\beta(t)<1$ for each $t \in K$. In particular, $d\left(y_{n}, y_{n+1}\right) \prec d\left(y_{n-1}, y_{n}\right)$ and so the sequence $\left\{d\left(y_{n}, y_{n+1}\right)\right\}$ is strictly decreasing (and bounded from below).

Since the cone $K$ is regular, there exists $\lim _{n \rightarrow \infty} d\left(y_{n}, y_{n+1}\right)=p$ and $\theta \preceq p \preceq d\left(y_{n}, y_{n+1}\right)$ for each $n$. Then $1>\beta(p)>\beta\left(d\left(y_{n}, y_{n+1}\right)\right)$ for each $n$, and hence

$$
d\left(y_{n}, y_{n+1}\right) \leq \beta(p) d\left(y_{n-1}, y_{n}\right) \preceq(\beta(p))^{2} d\left(y_{n-2}, y_{n-1}\right) \preceq \cdots \preceq(\beta(p))^{n} d\left(y_{0}, y_{1}\right),
$$

where $\beta(p)$ is a fixed scalar belonging to $[0,1)$.

Now we prove that $\left\{y_{n}\right\}$ is a Cauchy sequence in the usual way: for $m>n$ it is

$$
\begin{aligned}
d\left(y_{n}, y_{m}\right) & \leq d\left(y_{n}, y_{n+1}\right)+\cdots+d\left(y_{m-1}, y_{m}\right) \\
& \leq\left((\beta(p))^{n}+\cdots(\beta(p))^{m-1}\right) d\left(y_{0}, y_{1}\right) \\
& \leq \frac{(\beta(p))^{n}}{1-\beta(p)} d\left(y_{0}, y_{1}\right) \longrightarrow \theta \text { as } n \longrightarrow \infty
\end{aligned}
$$

Thus, by properties $\left(\mathrm{p}_{4}\right)$ and $\left(\mathrm{p}_{3}\right)$ and Definition $1.3,\left\{y_{n}\right\}$ is a Cauchy sequence in $g X$ and so there is $z \in X$ such that $f x_{n}=g x_{n+1} \rightarrow g z$ when $n \rightarrow \infty$. We will prove that $f z=g z$.

Put $x=x_{n}, y=z$ in the contractive condition. We obtain (writing temporarily $\alpha_{i}=$ $\left.\alpha_{i}\left(d\left(x_{n}, z\right)\right)\right)$ that

$$
\begin{aligned}
d\left(f x_{n}, f z\right) \leq & \alpha_{1} d\left(g x_{n}, g z\right)+\alpha_{2} d\left(g x_{n}, f x_{n}\right)+\alpha_{3} d(g z, f z) \\
& +\alpha_{4} d\left(f z, g x_{n}\right)+\alpha_{5} d\left(f x_{n}, g z\right) \\
\leq & \alpha_{1} d\left(g x_{n}, g z\right)+\left(\alpha_{2}+\alpha_{4}\right) d\left(g x_{n}, f x_{n}\right)+\alpha_{3} d(g z, f z) \\
& +\alpha_{4} d\left(f z, f x_{n}\right)+\alpha_{5} d\left(f x_{n}, g z\right) .
\end{aligned}
$$

Taking into account that all $\alpha_{i}{ }^{\prime}$ s are bounded in $[0,1)$ and that the abstract metric $d$ is continuous (because the cone $K$ is normal), passing to the limit in the last vector inequality, we obtain that

$$
d(g z, f z) \leq \alpha_{1} \cdot \theta+\left(\alpha_{2}+\alpha_{4}\right) \cdot \theta+\alpha_{3} d(g z, f z)+\alpha_{4} d(f z, g z)+\alpha_{5} \cdot \theta,
$$

that is, $d(g z, f z) \leq\left(\alpha_{3}+\alpha_{4}\right) d(g z, f z)$. Since $\alpha_{3}+\alpha_{4}<1$, it follows that $g z=f z=w$ and $f$ and $g$ have a point of coincidence $w$. 
Suppose that $w_{1}=f z_{1}=g z_{1}$ is another point of coincidence for $f$ and $g$. Then (3.10) implies that

$$
\begin{aligned}
d\left(w_{1}, w_{2}\right) & =d\left(f z, f z_{1}\right) \\
& \leq \alpha_{1} d\left(g z, g z_{1}\right)+\alpha_{2} d(g z, f z)+\alpha_{3} d\left(g z_{1}, f z_{1}\right)+\alpha_{4} d\left(f z_{1}, g z\right)+\alpha_{5} d\left(f z, g z_{1}\right) \\
& =\alpha_{1} d\left(w, w_{1}\right)+\alpha_{2} \cdot \theta+\alpha_{3} \cdot \theta+\alpha_{4} d\left(w_{1}, w\right)+\alpha_{5} d\left(w, w_{1}\right) \\
& =\left(\alpha_{1}+\alpha_{4}+\alpha_{5}\right) d\left(w, w_{1}\right) .
\end{aligned}
$$

Since $\alpha_{1}+\alpha_{4}+\alpha_{5}<1$, the last relation is possible only if $w=w_{1}$. So, the point of coincidence is unique.

If $(f, g)$ is weakly compatible, then [8, Proposition 1.4] implies that $f$ and $g$ have a unique common fixed point. The proof for the case in which $f X$ is complete is similar and is therefore omitted.

Remark 3.7. Taking $E=\mathbb{R}, K=[0,+\infty), g(x)=x$, we obtain a shorter proof of Theorem 1.6 (i.e., [28, Theorem 4]).

Remark 3.8. Taking appropriate choices of $f, g$ and $\alpha_{i}, i=1, \ldots, 5$ in Theorem 3.6, one can easily get the results of Reich (see relations (7), (8) in [28]), Hardy-Rogers (see relation (18) in [28]) and Ćirić (see relation (21) in [28]) in the setting of abstract metric spaces.

\section{Acknowledgments}

The authors (the first and the second) would like to acknowledge the financial support received from Universiti Kebangsaan Malaysia under the research Grant OUP-UKM-FST2012. The fourth and fifth authors are thankful to the Ministry of Science and Technological Development of Serbia.

\section{References}

[1] L. Kantorovitch, "The method of successive approximations for functional equations," Acta Mathematica, vol. 71, pp. 63-97, 1939.

[2] L. V. Kantorovič, "The principle of the majorant and Newton's method," vol. 76, pp. 17-20, 1951.

[3] J. S. Vandergraft, "Newton's method for convex operators in partially ordered spaces," SIAM Journal on Numerical Analysis, vol. 4, pp. 406-432, 1967.

[4] P. P. Zabreı̌ko, "K-metric and K-normed spaces: survey,” Collectanea Mathematica, vol. 48, pp. 852-859, 1997.

[5] K. Deimling, Nonlinear Functional Analysis, Springer, Berlin, Germany, 1985.

[6] H. Mohebi, "Topical functions and their properties in a class of ordered Banach spaces," in Continuous Optimization, Current Trends and Modern Applications, vol. 99, pp. 343-361, Springer, New York, NY, USA, 2005.

[7] L.-G. Huang and X. Zhang, "Cone metric spaces and fixed point theorems of contractive mappings," Journal of Mathematical Analysis and Applications, vol. 332, no. 2, pp. 1468-1476, 2007.

[8] M. Abbas and G. Jungck, "Common fixed point results for noncommuting mappings without continuity in cone metric spaces," Journal of Mathematical Analysis and Applications, vol. 341, no. 1, pp. 416-420, 2008. 
[9] M. Abbas and B. E. Rhoades, "Fixed and periodic point results in cone metric spaces," Applied Mathematics Letters, vol. 22, no. 4, pp. 511-515, 2009.

[10] M. Abbas, B. E. Rhoades, and T. Nazir, "Common fixed points for four maps in cone metric spaces," Applied Mathematics and Computation, vol. 216, no. 1, pp. 80-86, 2010.

[11] G. Jungck, S. Radenović, S. Radojević, and V. Rakočević, "Common fixed point theorems for weakly compatible pairs on cone metric spaces," Fixed Point Theory and Applications, Article ID 643840, 13 pages, 2009.

[12] S. Radenović, "Common fixed points under contractive conditions in cone metric spaces," Computers $\mathcal{E}$ Mathematics with Applications, vol. 58, no. 6, pp. 1273-1278, 2009.

[13] S. Radenović and B. E. Rhoades, "Fixed point theorem for two non-self mappings in cone metric spaces," Computers \& Mathematics with Applications, vol. 57, no. 10, pp. 1701-1707, 2009.

[14] S. Rezapour and R. Hamlbarani, "Some notes on the paper Cone metric spaces and fixed point theorems of contractive mappings," Journal of Mathematical Analysis and Applications, vol. 345, pp. 719-724, 2008.

[15] W.-S. Du, "A note on cone metric fixed point theory and its equivalence," Nonlinear Analysis: Theory, Methods \& Applications, vol. 72, no. 5, pp. 2259-2261, 2010.

[16] Z. Kadelburg, S. Radenović, and V. Rakočević, "Topological vector space-valued cone metric spaces and fixed point theorems," Fixed Point Theory and Applications, Article ID 170253, 18 pages, 2010.

[17] S. Janković, Z. Kadelburg, and S. Radenović, “On cone metric spaces: a survey," Nonlinear Analysis: Theory, Methods \& Applications, vol. 74, no. 7, pp. 2591-2601, 2011.

[18] M. A. Krasnoseljski and P. P. Zabrejko, Geometrical Methods in Nonlinear Analysis, Springer, 1984.

[19] M. G. KreĬn and M. A. Rutman, "Linear operators leaving invariant a cone in a Banach space," Akademiya Nauk SSSR i Moskovskoe Matematicheskoe Obshchestvo, vol. 3, no. 1, pp. 3-95, 1948.

[20] Y. J. Cho, B. E. Rhoades, R. Saadati, B. Samet, and W. Shantawi, "Nonlinear coupled fixed point theorems in ordered generalized metric spaces with integral type," Fixed Point Theory and Applications, vol. 2012, article 8, 2012.

[21] Y. J. Cho, R. Saadati, and S. Wang, "Common fixed point theorems on generalized distance in ordered cone metric spaces," Computers E Mathematics with Applications, vol. 61, no. 4, pp. 1254-1260, 2011.

[22] E. Graily, S. M. Vaezpour, R. Saadati, and Y. J. Cho, “Generalization of fixed point theorems in ordered metric spaces concerning generalized distance," Fixed Point Theory and Applications, vol. 2011, article 30, 2011.

[23] Z. M. Fadail, A. G. B. Ahmad, and Z. Golubović, "Fixed foint theorems of single-valued mapping for c-distance in cone metric spaces," Abstract and Applied Analysis, vol. 2012, Article ID 826815, 11 pages, 2012.

[24] Z. M. Fadail and A. G. B. Ahmad, "Coupled fixed foint theorems of single-valued mapping for cdistance in cone metric spaces," Journal of Applied Mathematics, vol. 2012, Article ID 246516, 20 pages, 2012.

[25] Z. M. Fadail and A. G. B. Ahmad, "Common coupled fixed foint theorems of single-valued mapping for c-distance in cone metric spaces," Abstract and Applied Analysis, vol. 2012, Article ID 901792, 24 pages, 2012.

[26] Z. M. Fadail, A. G. B. Ahmad, and L. Paunović, "New fixed point results of single-valued mapping for c-distance in cone metric spaces," Abstract and Applied Analysis, vol. 2012, Article ID 639713, 12 pages, 2012.

[27] A. Singh, R. C. Dimri, and S. Bhatt, "A unique common fixed point theorem for four maps in cone metric spaces," International Journal of Mathematical Analysis, vol. 4, no. 29-32, pp. 1511-1517, 2010.

[28] B. E. Rhoades, "A comparison of various definitions of contractive mappings," Transactions of the American Mathematical Society, vol. 226, pp. 257-290, 1977.

[29] G. S. Jeong and B. E. Rhoades, "Maps for which $F(T)=F\left(T^{n}\right)$," Fixed Point Theory and Applications, vol. 6, pp. 87-131, 2005.

[30] A. Amini-Harandi and M. Fakhar, "Fixed point theory in cone metric spaces obtained via the scalarization method," Computers E Mathematics with Applications, vol. 59, no. 11, pp. 3529-3534, 2010.

[31] Z. Kadelburg, S. Radenović, and V. Rakočević, "A note on the equivalence of some metric and cone metric fixed point results," Applied Mathematics Letters, vol. 24, no. 3, pp. 370-374, 2011. 


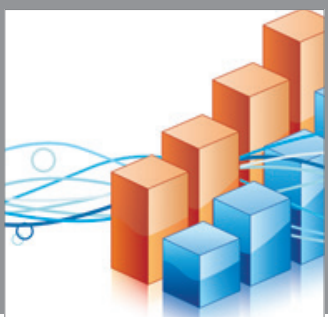

Advances in

Operations Research

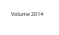

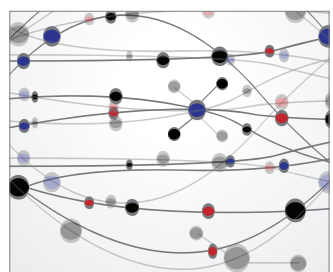

\section{The Scientific} World Journal
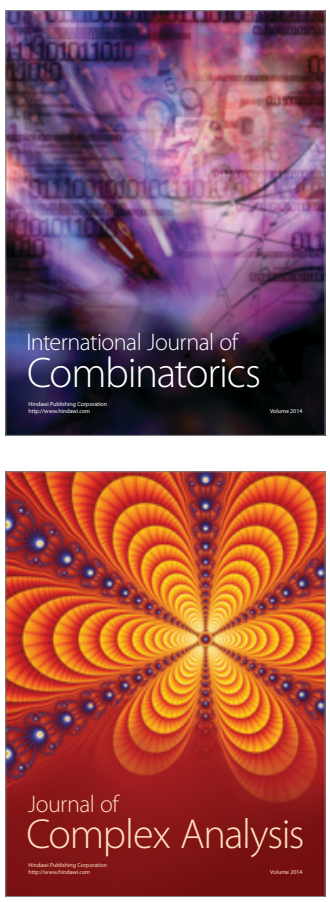

International Journal of

Mathematics and

Mathematical

Sciences
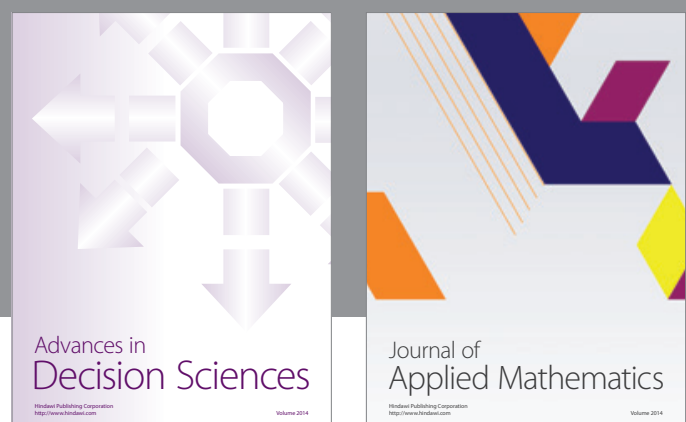

Journal of

Applied Mathematics
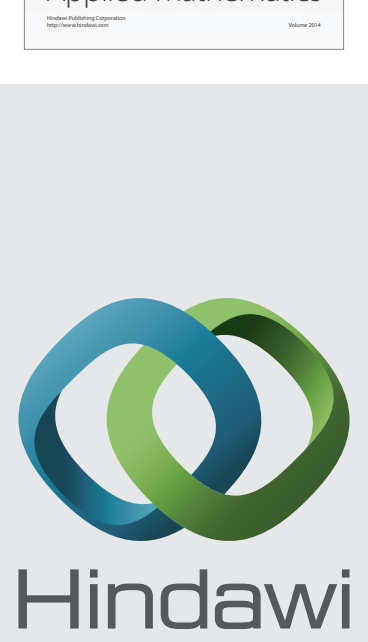

Submit your manuscripts at http://www.hindawi.com
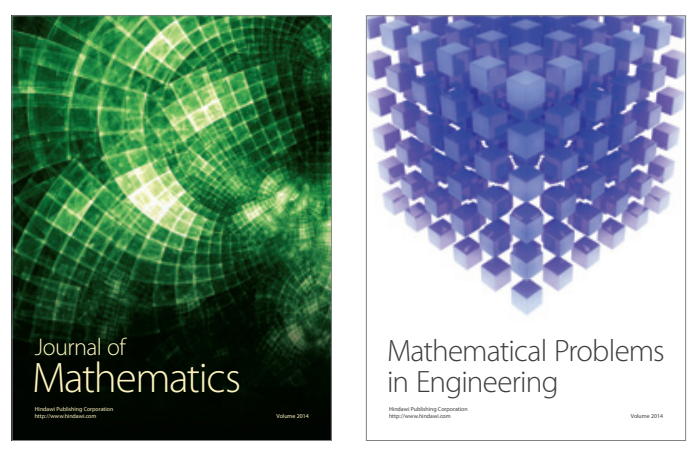

Mathematical Problems in Engineering
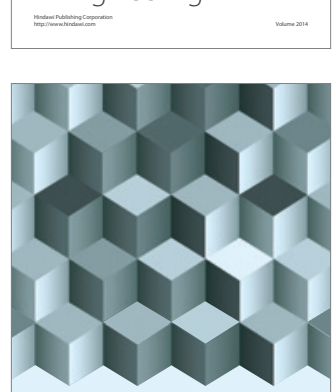

Journal of

Function Spaces
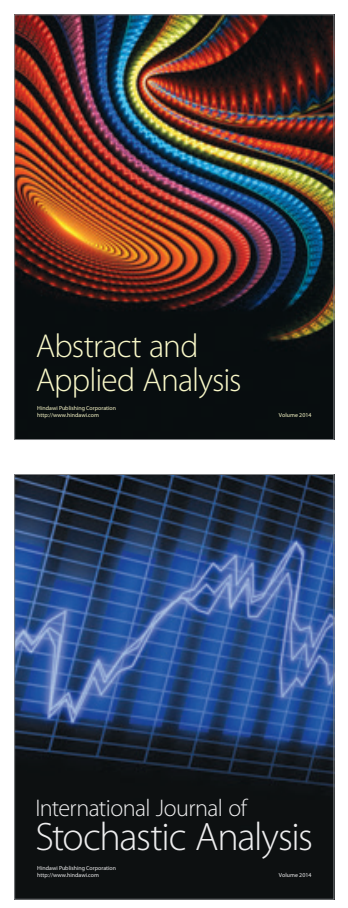

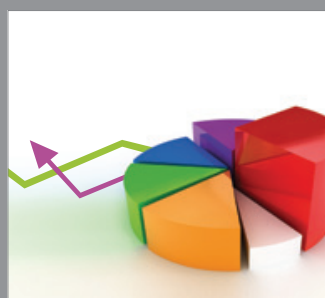

ournal of

Probability and Statistics

Promensencen
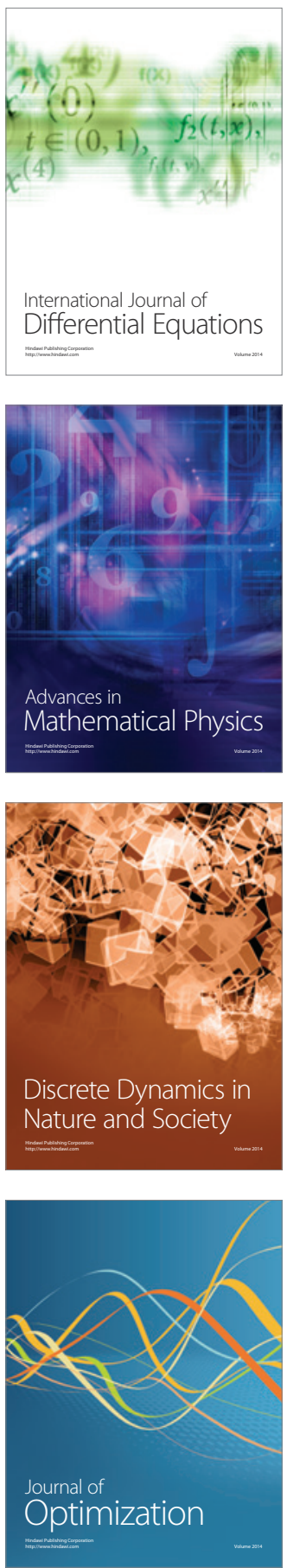